\title{
Coupling-Matrix Design of Dual and Triple Passband Filters
}

\author{
Marjan Mokhtaari, Jens Bornemann, Fellow, IEEE, K. Rambabu, and Smain Amari, Member, IEEE
}

\begin{abstract}
The concept of the conventional coupling matrix is extended to include designs of dual- and triple-band filters. The multiband response is created by either placing transmission zeros within the bandwidth of a wideband filter or using higher order resonances. Realizable topologies both in planar and waveguide technologies can be imposed and associated coupling coefficients enforced during optimization. The design process is verified by measurements and comparison with results of commercially available field solvers.
\end{abstract}

Index Terms-Dual-band filters, filters, microstrip filters, waveguide filters.

\section{INTRODUCTION}

$\mathbf{R}$ ECENT developments in microwave filters have focused on many different topics, one of them being the design of dual- and triple-band filters. They are in heavy demand due to applications in modern wireless and satellite systems. Usually, theoretical design techniques are related to individual filter technologies. For instance, lumped-element or stepped-impedances approaches are used in low-temperature co-fired ceramic (LTCC) applications, e.g., [1], [2]. So-called dual-behavior resonators [3], [4] create attenuation poles at specific frequencies in order to separate individual passbands. The coupling between two modified open-loop resonators is used to create a dual-band filter in microstrip technology [5]. Polynomial approaches and coupling matrices are applied to the design of dual-band bandpass [6] and bandstop filters [7] in waveguide technology.

Common to all such design procedures is the fact that they cannot immediately be used if the filter topology changes. Moreover, many approaches are limited with respect to the number of transmission zeros and locations over the frequency band of interest.

Therefore, this paper focuses on the design of dual- and tripleband filters by employing the coupling matrix and the optimization of its entries. One of the major advantages of this approach is that topologies and certain coupling elements can be controlled from the onset [8]. The basic approach of this method was introduced in [9]. However, measurements failed to confirm the transmission zeros between individual passbands.

Manuscript received March 22, 2006; revised August 3, 2006.

M. Mokhtaari and J. Bornemann are with the Department of Electrical and Computer Engineering, University of Victoria, Victoria, BC, Canada V8W 3P6.

K. Rambabu is with the Institute for Infocomm Research, Singapore 117674.

S. Amari is with the Department of Electrical and Computer Engineering,

Royal Military College of Canada, Kingston, ON, Canada K7K 7B4.

Color versions of Figs. 3, 4, 5(a), and 6(a) are available online at http://ieeexplore.ieee.org.

Digital Object Identifier 10.1109/TMTT.2006.884687
In this paper, we present a new design and measurements, which validate the design approach in microstrip technology. Moreover, the same theory is applied to dual-band waveguide filters and shows that the inclusion of higher order mode resonances aids in the design. It is thus demonstrated that the design process is generally applicable to multiband filter designs in varying topologies and technologies.

We are using hairpin resonators [10] and open-loop resonators [11] as triple/dual-band examples in microstrip technology. Dual-band waveguide designs include folded cavity filters, e.g., [12], and inline dual-mode configurations, e.g., [13], [14]. Folded waveguide filters make use of higher order resonances in addition to the dual-band design, whereas the dual-band effect in inline configurations is created because of higher order resonances.

\section{Design PROCEDURE}

The basic approach to create a coupling matrix for given specifications of a single bandpass filter is presented in [8] and [15]. (The reader is referred to [8] and [15] for further details.) The principal advantages of this technique are, first, that the topology of any scheme of coupled resonators can be specified in advance and, secondly, that the signs and limits of coupling coefficients can be strictly enforced during optimization.

For the design of dual- and triple-band filters, we assume first that a single wideband filter will be constructed whose bandwidth covers all bandwidths of the dual- and/or triple-band filters. Since the maximum number $N$ of realizable transmission zeros is dictated by the topology, any number $n \leq N$ of transmission zeros can now be placed within the initial broad passband in order to separate individual passbands. Optimization [15] is then employed to adjust the entries of the coupling matrix.

The basic design steps are shown here at the example of a triple-band filter with six resonators and four transmission zeros, each two of which are located between adjacent passbands. The individual passbands are centered at $2.65,3$, and $3.35 \mathrm{GHz}$, and more than $50-\mathrm{MHz}$ bandwidth is to be retained in each of the bands.

We first design a standard Chebyshev response over the entire triple-band frequency range using $3 \mathrm{GHz}$ as the center frequency, 800-MHz bandwidth, and 24-dB return loss. This design is shown in Fig. 1 as dashed lines. To allow a coupling matrix to be optimized, we then require, first, a prototype function for the triple-band filter and, secondly, an initial coupling matrix to start the optimization. One possibility to obtain the prototype function is to optimize the coefficients of the numerator and denominator of the filtering function. This is usually done 


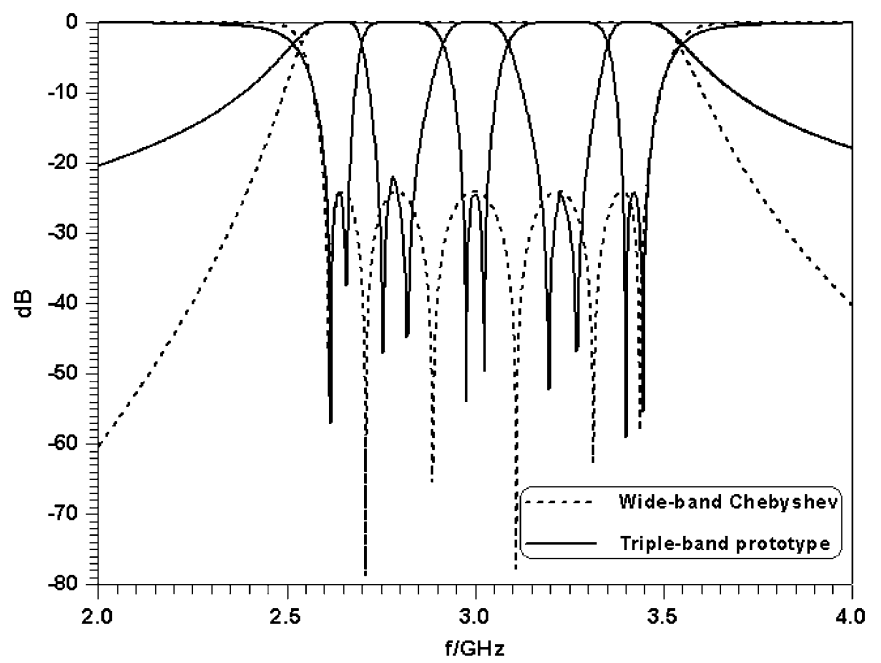

Fig. 1. Wideband Chebyshev and triple-band prototype responses of design example.

to determine the prototype function and the positions of the reflection and transmission zeros in particular. Our approach is different though. We obtain the prototype function from piecing together the functions of three individual filters, each of which is designed according to the single bandpass filter approach in [15]. For the triple-band filter considered in this example, the left-most response is that of a two-pole filter with a transmission zero to the right, the center response corresponds to a two-pole elliptic-function filter (one transmission zero on each side), and the right-most response is that of a two-pole filter with one transmission zero on the left. The so-obtained overall prototype function is shown in Fig. 1 as solid lines. The three different parts are clearly distinguished by the discontinuous points between the passbands at 2.78 and $3.23 \mathrm{GHz}$.
The choice of an initial coupling matrix involves the actual circuit topology. Let us assume that the triple-band filter be designed for microstrip technology on RT6006 substrate and that six hairpin resonators be employed. The four transmission zeros are to be created by cross couplings between hairpin resonators 1 and 6 , as well as 2 and 5, thus specifying a symmetric folded filter configuration. One of the simplest initial coupling matrices is that of the standard wideband Chebyshev filter (cf. Fig. 1, dashed line) and allowing for the additional cross couplings 1-6 and $2-5$. As the sign of the coupling is determined by the orientation of the hairpin resonators, we allow both magnetic and electric couplings in the optimization. For the given example, a quick calculation using the closed-form expressions in [16] advises that the magnitude of normalized inline coupling coefficients be less that 0.9 and that of the cross couplings be less than 0.5 . With this initial coupling matrix, the optimization produces the following coupling matrix (including source and load), as shown in (1) at the bottom of this page, whose performance is shown as dashed lines in Fig. 2. Depending on the initial values, different coupling matrices are obtained. For instance, using direct and cross couplings to be 0.5 and 0.25 in magnitude, respectively, and specifying two couplings $M_{12}$ and $M_{25}$ as negative, a different final matrix is obtained, as shown in (2) at the bottom of this page, and its performance is shown as solid lines in Fig. 2. Note that both approaches result in a very small coupling between resonators 3 and 4; it is actually much smaller than 0.0001 in (2) and, therefore, appears as zero.

Whereas both matrices $M_{1}$ and $M_{2}$ adhere to the restrictions specified above, the filter governed by $M_{2}$ is attractive due to the fact that one of the coupling coefficients $M_{34}$ vanishes. A disadvantage compared to that given by $M_{1}$ is the reduced return loss in the center band. This filter can now be designed by translating the coupling coefficients into line dimensions on an RT6006 substrate using commercial field solvers. The Ansoft

$$
M_{1}=\left[\begin{array}{cccccccc}
0 & 0.6541 & 0 & 0 & 0 & 0 & 0 & 0 \\
0.6541 & 0 & 0.7689 & 0 & 0 & 0 & 0.4792 & 0 \\
0 & 0.7689 & 0 & 0.5740 & 0 & -0.0112 & 0 & 0 \\
0 & 0 & 0.5740 & 0 & -0.0157 & 0 & 0 & 0 \\
0 & 0 & 0 & -0.0157 & 0 & 0.5740 & 0 & 0 \\
0 & 0 & -0.0112 & 0 & 0.5740 & 0 & 0.7689 & 0 \\
0 & 0.4792 & 0 & 0 & 0 & 0.7689 & 0 & 0.6541 \\
0 & 0 & 0 & 0 & 0 & 0 & 0.6541 & 0
\end{array}\right]
$$

$$
M_{2}=\left[\begin{array}{cccccccc}
0 & 0.6251 & 0 & 0 & 0 & 0 & 0 & 0 \\
0.6251 & 0 & -0.8128 & 0 & 0 & 0 & 0.4450 & 0 \\
0 & -0.8128 & 0 & 0.5488 & 0 & -0.0200 & 0 & 0 \\
0 & 0 & 0.5488 & 0 & 0 & 0 & 0 & 0 \\
0 & 0 & 0 & 0 & 0 & 0.5488 & 0 & 0 \\
0 & 0 & -0.0200 & 0 & 0.5488 & 0 & -0.8128 & 0 \\
0 & 0.4450 & 0 & 0 & 0 & -0.8128 & 0 & 0.6251 \\
0 & 0 & 0 & 0 & 0 & 0 & 0.6251 & 0
\end{array}\right]
$$




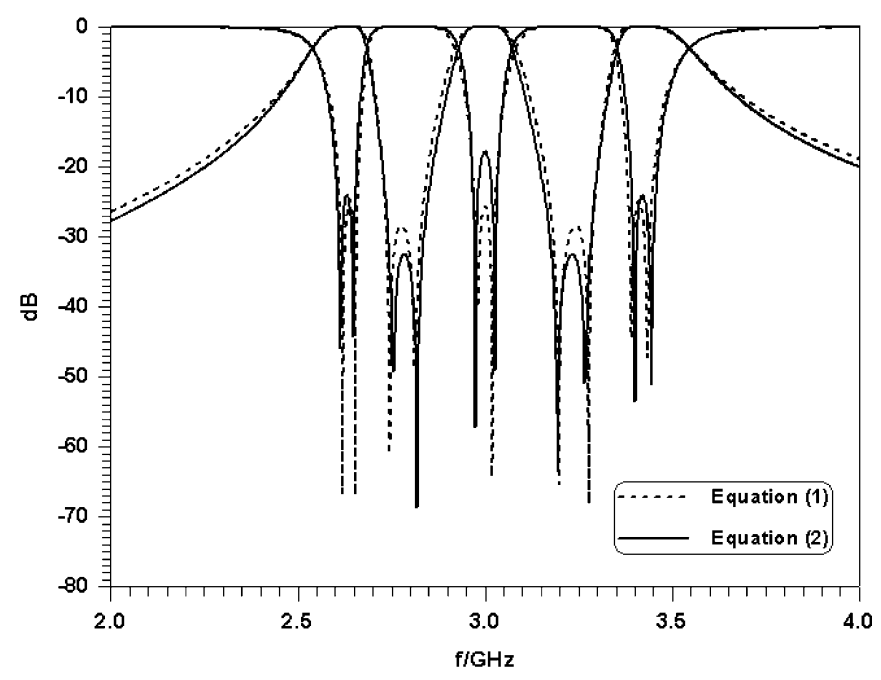

Fig. 2. Responses of triple-band coupling matrices obtained by optimization from different initial values.

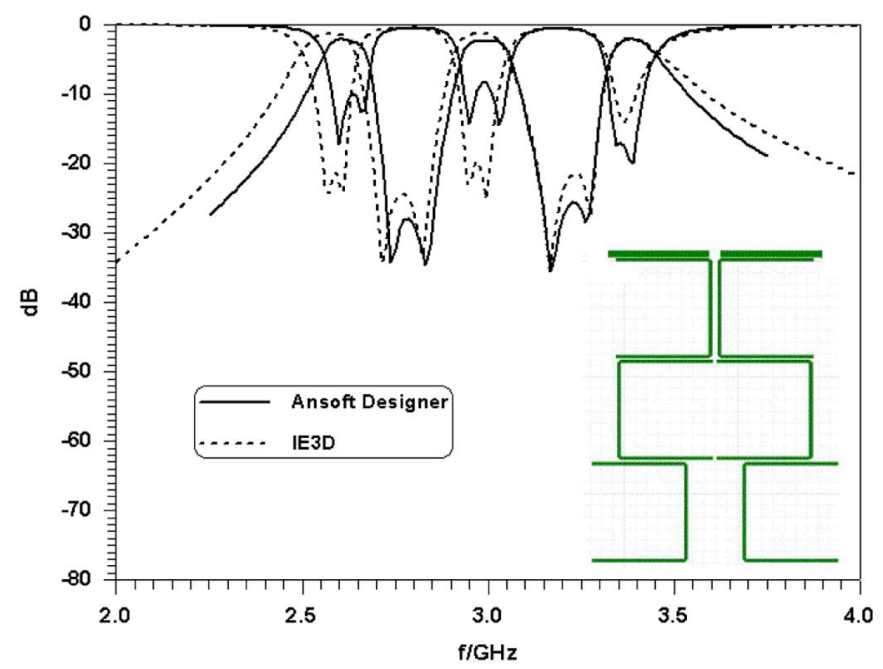

Fig. 3. Performance of triple-band filter using hairpin resonators on RT 6006 substrate. Ansoft Designer (solid lines, data from [9]) and IE3D (dotted lines).

Designer response (solid lines in Fig. 3) is obtained by equating the coupling coefficients of (2) with those in the actual structure (inset of Fig. 3). Of course, the achievable precision is limited in this step due to comparison of an equivalent circuit (coupling matrix) with a full-wave electromagnetic (EM) model. Therefore, slight differences between responses of the coupling matrix and that of the actual circuit must be accepted. Some minor fine-tuning in the EM-based code is also usually required. The design is verified by a different commercial package, i.e., IE3D, whose response is denoted as dashed lines in Fig. 3. (Note that slight differences between Ansoft Designer and IE3D results have been previously observed [17] and are attributed to the different approaches of the method-of-moments implementation in both packages.) The passband insertion losses, as simulated with Ansoft Designer, are approximately 2.0, 2.3 and $2.1 \mathrm{~dB}$; the minimum passband return loss is slightly below $10 \mathrm{~dB}$.

Depending on the actual filter topology and technology, tighter restrictions might have to be imposed on magnitudes and signs of individual coupling coefficients. For planar circuits, closed-form expressions for the electric and magnetic coupling coefficients of, for example, open-loop resonators [16], are used. For waveguide-based filters, maximum aperture dimensions are determined in advance and related coupling coefficients are calculated from simple mode-matching routines, e.g., [18].

\section{RESULTS}

The above theory is now applied to dual-band filters in microstrip and waveguide technologies. A few microstrip examples are presented in [9] and will not be repeated here. However, due to manufacturing tolerances, the transmission zeros between passbands are not experimentally confirmed in [9]. Therefore, a six-resonator dual-band microstrip filter with open-loop resonators is designed on an RT5880 substrate with a height of $508 \mu \mathrm{m}$. The design parameters are $f_{0}=3 \mathrm{GHz}$ and $b=500 \mathrm{MHz}$ and the transmission zeros at 2.64, 2.94, 3.06 , and $3.42 \mathrm{GHz}$. The initial coupling values in the coupling scheme shown in the inset of Fig. 4(a) are all 0.5, except for $M_{16}=-0.2$. The optimized coupling matrix is given in (3), as shown at the bottom of this page. Fig. 4(a) shows the comparison between the performances of the coupling matrix and that of the actual circuits using Ansoft Designer. A prototype filter was built, and its response is also shown in Fig. 4(b) together with a photograph and the Ansoft Designer data for comparison.

The measurements confirm the basic shape of the computed filter characteristic, especially the existence of the four transmission zeros. However, the entire measurement is slightly shifted towards higher frequencies. After investigation, it was determined that this shift can be partly attributed to the fact that along the tracks (transmissions lines), the manufacturing process produces slightly deeper cuts into the dielectric. Therefore, the ef-

$$
M_{3}=\left[\begin{array}{cccccccc}
0 & 0.8719 & 0 & 0 & 0 & 0 & 0 & 0 \\
0.8719 & 0 & 0.7001 & 0.6005 & 0 & 0 & -0.2373 & 0 \\
0 & 0.7001 & 0 & 0 & 0 & 0.0700 & 0 & 0 \\
0 & 0.6005 & 0 & 0 & 0.9440 & 0 & 0 & 0 \\
0 & 0 & 0 & 0.9440 & 0 & 0 & 0.6005 & 0 \\
0 & 0 & 0.0700 & 0 & 0 & 0 & 0.6986 & 0 \\
0 & -0.2373 & 0 & 0 & 0.6005 & 0.6986 & 0 & 0.8719 \\
0 & 0 & 0 & 0 & 0 & 0 & 0.8719 & 0
\end{array}\right]
$$




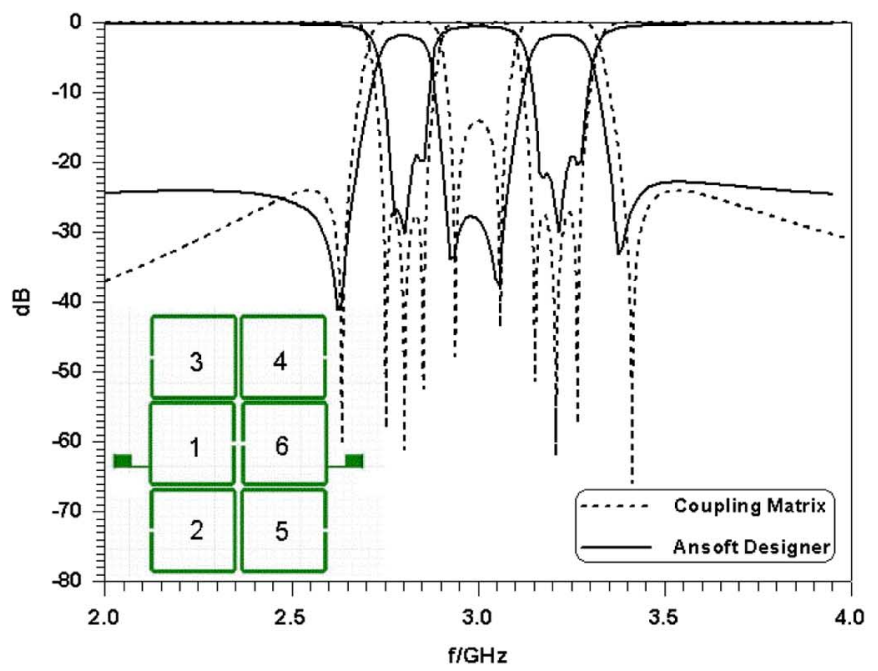

(a)

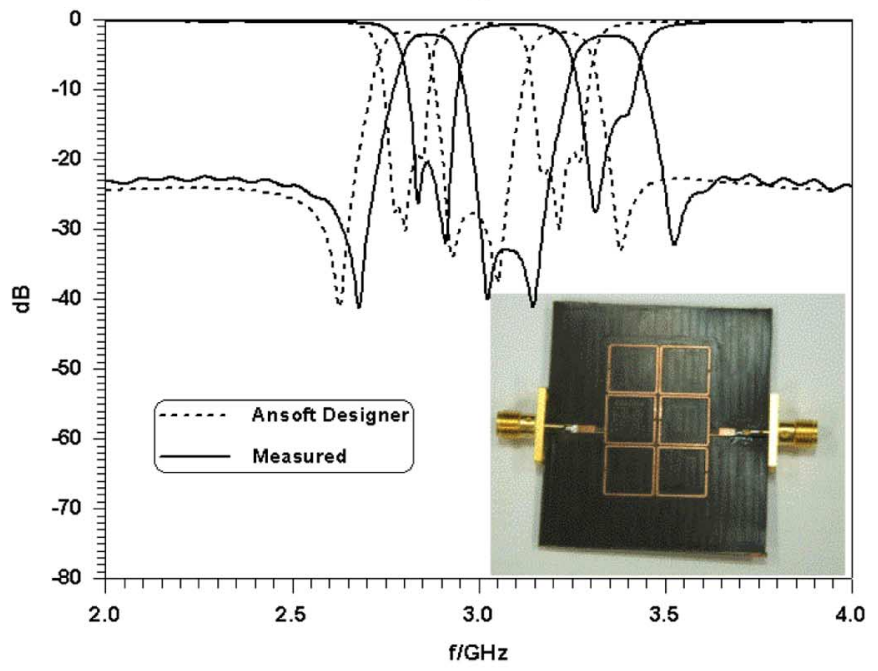

(b)

Fig. 4. Performance of a dual-band filter using six open-loop resonators on RT 5880 substrate. (a) Comparison of coupling-matrix theory (dashed lines) with Ansoft Designer (solid lines). (b) Prototype measurements (solid lines) and photograph (Ansoft Designer data (dashed lines) for comparison).

fective dielectric constant of the line will be lower than expected. Note that this effect is more pronounced for the thin lines used to form the open-loop resonators whose linewidths are much smaller than those of the 50- $\Omega$ input and output sections. The remaining discrepancies are attributed to manufacturing tolerances, which compare well with measurements presented in [9] for higher permittivity substrates. Measured passband insertion losses are 2.1 and $2.4 \mathrm{~dB}$.

Single passband waveguide filters are traditionally modeled by coupling matrices, e.g., [19]. We are extending the dual-band design of Section II to waveguide technology by making use of two principles. The first example uses the same procedure as applied to the microstrip filters, but takes into account additional transmission zeros produced by the actual filter. In the second example, we will use higher order resonances in an inline configuration to create the second passband. This principle was first applied in [13].

Fig. 5(a) shows a dual-band filter in folded waveguide technology. The design parameters are $f_{0}=15 \mathrm{GHz}$ and $b=2$

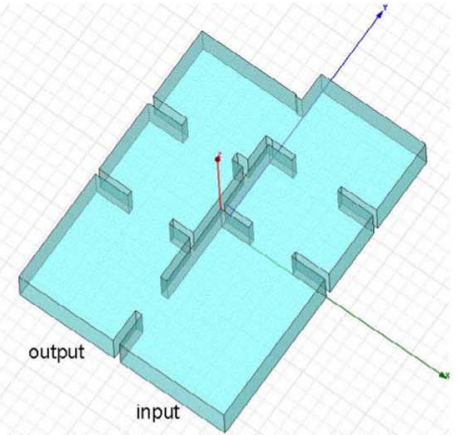

(a)

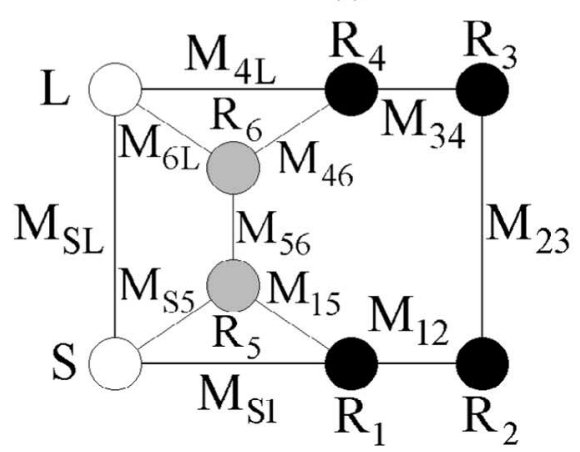

(b)

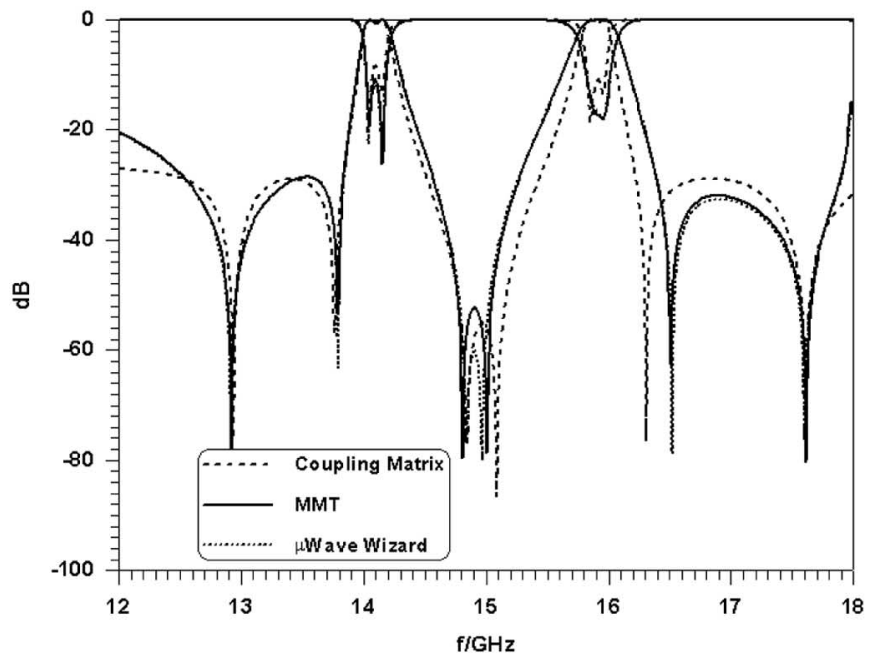

(c)

Fig. 5. Dual-band filter in folded waveguide technology. (a) Three-dimensional (3-D) view of filter. (b) Coupling scheme. (c) Performances obtained from theory (dashed lines) and EM-based software (solid and dotted lines).

$\mathrm{GHz}$ and transmission zeros at 12.94, 14.85, 15.09, and 16.31 $\mathrm{GHz}$. In order to convert the optimized coupling matrix elements to actual aperture dimensions, we follow an approach given in [12]. Upon inspection of a first waveguide design, additional transmission zeros were observed resulting from the distance between the source/load aperture and the rest of the filter. The two resonances were included in the coupling scheme [see Fig. 5(b)] as detuned nodes $R_{5}$ and $R_{6}$ similar to [20]. The optimized coupling matrix is shown in (4) at the bottom of the following page, and its response is shown via dashed lines in Fig. 5(c). Good agreement is obtained with the actual filter as modeled by the mode-matching technique (MMT) (solid lines) 
and the $\mu$ WaveWizard (dotted lines), especially with respect to the location of the two passbands and the number of transmission zeros.

Fig. 6(a) shows an inline dual-mode filter using $\mathrm{TE}_{101}$ and $\mathrm{TE}_{011}$ modes in the two cavities. This type of filter was introduced in [14]. Here we present a design for dual-band operation at 10.5 and $11.75 \mathrm{GHz}$. The second passband results from the next higher order modes in the two cavities. In the coupling scheme of Fig. 6(b), they are modeled as detuned resonators $R_{3}$ and $R_{4}$ in an attempt to maintain symmetry of the coupling matrix. Note that this design differs from all previous ones in this paper due to the fact that the second (higher) passband cannot be controlled independently. The higher order resonances depend on the two cavities, whose dimensions are determined to obtain the first (lower) passband. Therefore, the midband frequency and bandwidth in this design are those of the lower passband ( $f_{0}=10.5 \mathrm{GHz}, b=210 \mathrm{MHz}$ ). In the coupling scheme [see Fig. 6(b)], the straight line from input to output including 1-6 cross-coupling forms a standard quadruplet for the lower frequency band. The addition and connection of the higher order resonances in Fig. 6(b) are based on the following considerations.

In the first section of the dual-mode resonators, $\mathrm{TE}_{11}$ and $\mathrm{TM}_{11}$ are well below cutoff even in the upper frequency band. In the second (larger) section of the dual-mode resonators, both $\mathrm{TE}_{11}$ and $\mathrm{TM}_{11}$ are above cutoff. However, this section is too short for a $\mathrm{TE}_{111}$ resonance to occur and, therefore, nodes $R_{3}$ and $R_{4}$ represent $\mathrm{TM}_{110}$ resonances. Therefore, both fundamental resonances $\mathrm{TE}_{101}$ and $\mathrm{TE}_{011}$ couple to the $\mathrm{TM}_{110}$ higher order resonance. Above $11.4 \mathrm{GHz}$, the input/output irises become propagating. That means that the input/output can couple directly to the $\mathrm{TM}_{110}$ resonances $R_{3} / R_{4}$. Since the center iris of the filter is centered with respect to the two adjacent cavities, there is no $\mathrm{TE}_{10 / 01}-\mathrm{TM}_{11}$ coupling through the center iris, i.e., $M_{24}=M_{14}=M_{35}=M_{36}=0$ ).

The coupling matrix obtained from following this scheme is given in (5). Its response is shown in Fig. 6(c) via dashed lines. The actual design as computed with the High-Frequency Structure Simulator (HFSS) (dotted lines) and the coupled integral-equation technique (CIET) (solid lines) agrees relatively well with the coupling-matrix prediction, shown in (5) at the top of the following page.

Except for the small deviations between coupling matrix and full-wave codes addressed earlier, the only notable discrepancy is the location of the transmission zero between $11-11.5 \mathrm{GHz}$.

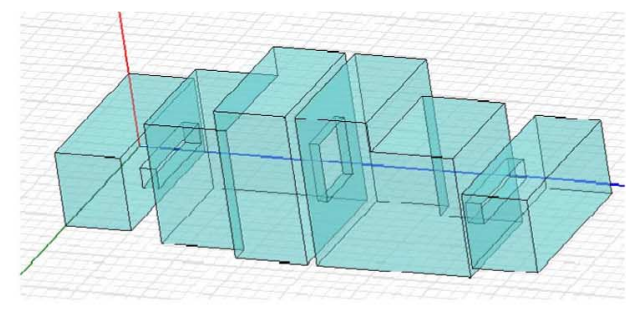

(a)

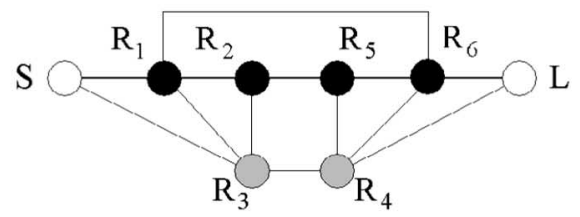

(b)

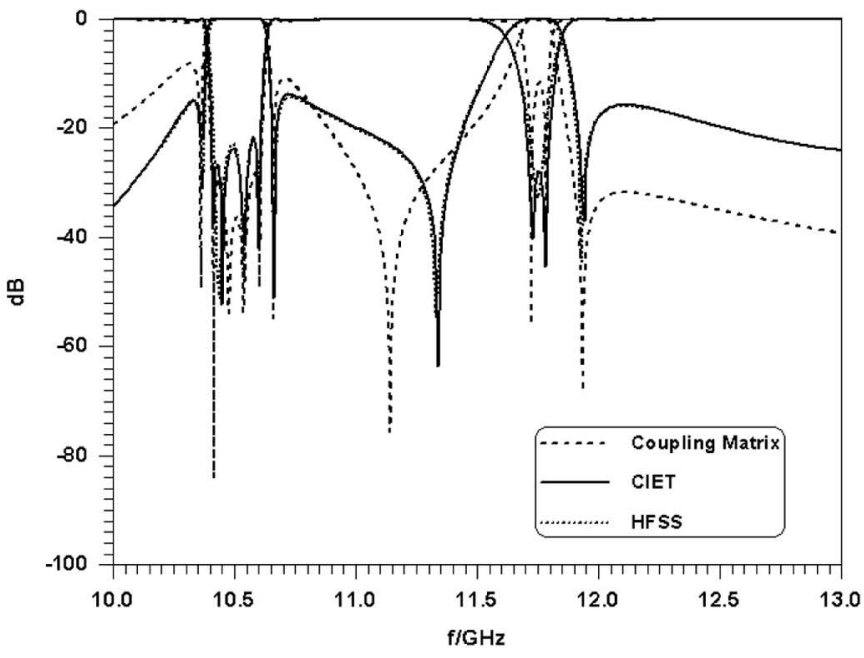

(c)

Fig. 6. Inline dual-band waveguide filter based on fundamental and higher order mode resonances. (a) 3-D-view of filter. (b) Coupling scheme. (c) Performances obtained from theory (dashed lines) and EM-based software (solid and dotted lines, which are almost indistinguishable).

This is attributed to the fact that the $\mathrm{TM}_{11}-\mathrm{TM}_{11}$ coupling through the center iris is highly frequency dependent in this frequency range. Such dependence is, of course, not captured by a coupling matrix approach, which assumes constant coupling coefficients. This is a limitation not only of this coupling-matrix design procedure, but of other coupling matrix designs as well. Nevertheless, this example demonstrates the flexibility of the coupling-matrix design routine presented in this paper.

$$
M_{4}=\left[\begin{array}{cccccccc}
0 & 0.3477 & 0 & 0 & 0 & 0.6000 & 0 & -0.0242 \\
0.3477 & 0.0136 & 0.9000 & 0 & 0 & 0.0500 & 0 & 0 \\
0 & 0.9000 & 0.0407 & 0.1588 & 0 & 0 & 0 & 0 \\
0 & 0 & 0.1588 & 0.0407 & 0.9000 & 0 & 0 & 0 \\
0 & 0 & 0 & 0.9000 & 0.0136 & 0 & 0.0500 & 0.3477 \\
0.6000 & 0.0500 & 0 & 0 & 0 & 2.2000 & 0.0050 & 0 \\
0 & 0 & 0 & 0 & 0.0500 & 0.0050 & -2.4000 & 0.7000 \\
-0.0242 & 0 & 0 & 0 & 0.3477 & 0 & 0.7000 & 0
\end{array}\right]
$$




$$
M_{5}=\left[\begin{array}{cccccccc}
0 & 1.3410 & 0 & 0.0024 & 0 & 0 & 0 & 0 \\
1.3410 & -1.9990 & 0.7752 & 4.2254 & 0 & 0 & -1.0767 & 0 \\
0 & 0.7752 & -0.0884 & 0.3869 & 0 & 1.0093 & 0 & 0 \\
0.0024 & 4.2254 & 0.3869 & -9.3966 & 0.6955 & 0 & 0 & 0 \\
0 & 0 & 0 & 0.6955 & -9.3966 & 0.3869 & 4.2254 & 0.0024 \\
0 & 0 & 1.0093 & 0 & 0.3869 & -0.0884 & 0.7752 & 0 \\
0 & -1.0767 & 0 & 0 & 4.2254 & 0.7752 & -1.9990 & 1.3410 \\
0 & 0 & 0 & 0 & 0.0024 & 0 & 1.3410 & 0
\end{array}\right]
$$

Note that the publication of all dimensions of the filters presented in this paper is prohibitive due to lack of space. Please contact the first author for actual dimensions of individual filters.

\section{CONCLUSION}

The coupling-matrix design procedure for dual- and triplepassband filters presents a viable alternative to current multiband filter design techniques. The method is flexible, not restricted to certain filter types or topologies, and is capable of including higher order mode effects. The coupling scheme and limitations for the coupling coefficients can be enforced during the design. The feasibility of this technique is demonstrated for filter configurations in microstrip and waveguide technology. The designs are verified by measurements and a number of different full-wave codes.

\section{REFERENCES}

[1] Y.-X. Guo, L. C. Ong, M. Y. W. Chia, and B. Luo, "Dual-band bandpass filter in LTCC," in IEEE MTT-S Int. Microw. Symp. Dig., Long Beach, Jun. 2005, pp. 2219-2222.

[2] H. Miyake, S. Kitazawa, T. Ishizaki, T. Yamanda, and Y. Nagatomi, "A miniaturized monolithic dual-band filter using ceramic lamination technique for dual-mode portable telephones," in IEEE MTT-S Int. Microw. Symp. Dig., Denver, Jun. 1997, pp. 789-792.

[3] C. Quendo, E. Rius, and C. Person, "Narrow bandpass filters using dual-behavior resonators," IEEE Trans. Microw. Theory Tech., vol. 51, no. 3, pp. 734-743, Mar. 2003.

[4] C. Quendo, E. Ruis, and C. Person, "An original topology of dual-band filter with transmission zeros," in IEEE MTT-S Int. Microw. Symp. Dig., Philadelphia, PA, Jun. 2003, pp. 1093-1096.

[5] M.-L. Chuang, "Concurrent dual band filter using single set of microstrip open-loop resonators," Electron. Lett., vol. 41, pp. 1013-1014, Sep. 2005.

[6] Macchiarella and S. Tamiazzo, "Design techniques for dual-passband filters," IEEE Trans. Microw. Theory Tech., vol. 53, no. 11, pp. 3265-3271, Nov. 2005.

[7] R. J. Cameron, M. Yu, and Y. Wang, "Direct-coupled microwave filters with single and dual stopbands," IEEE Trans. Microw. Theory Tech., vol. 53, no. 11, pp. 3288-3297, Nov. 2005.

[8] S. Amari, U. Rosenberg, and J. Bornemann, "Adaptive synthesis and design of resonator filters with source/load-multiresonator coupling," IEEE Trans. Microw. Theory Tech., vol. 50, no. 8, pp. 1969-1978, Aug. 2002.

[9] M. Mokhtaari, J. Bornemann, and S. Amari, "Coupling-matrix design of dual/triple-band uni-planar filters," in IEEE MTT-S Int. Microw. Symp. Dig., San Francisco, CA, Jun. 2006, pp. 515-518.

[10] E. G. Cristal and S. Frankel, "Hair-pin line and hybrid hair-pin line/ half-wave parallel-coupled-line filters," IEEE Trans. Microw. Theory Tech., vol. MTT-20, no. 11, pp. 719-728, Nov. 1972.

[11] J. S. Hong and M. J. Lancaster, "Aperture-coupled microstrip openloop resonators and their applications to the design of novel microstrip bandpass filters," IEEE Trans. Microw. Theory Tech., vol. 47, no. 9, pp. 1848-1855, Sep. 1999.

[12] J. Bornemann, S. Amari, and R. Vahldieck, "A flexible $S$-matrix algorithm for the design of folded waveguide filters," in Proc. 35th Eur. Microw. Conf., Paris, France, Oct. 2005, pp. 405-408.
[13] U. Rosenberg, "Multiplexing and double band filtering with commonmultimode cavities," IEEE Trans. Microw. Theory Tech., vol. 38, no. 12, pp. 1862-1871, Dec. 1990.

[14] J. Bornemann, U. Rosenberg, S. Amari, and R. Vahldieck, "Edge-conditioned vector basis functions for the analysis and optimization of rectangular waveguide dual-mode filters," in IEEE MTT-S Int. Microw. Symp. Dig., Anaheim, CA, Jun. 1999, pp. 1695-1698.

[15] S. Amari, "Synthesis of cross-coupled resonator filters using an analytical gradient-based optimization technique," IEEE Trans. Microw. Theory Tech., vol. 48, no. 9, pp. 1559-1564, Sep. 2000.

[16] J. S. Hong and M. J. Lancaster, "Coupling of microstrip square open-loop resonators for cross-coupled planar microwave filters," IEEE Trans. Microw. Theory Tech., vol. 44, no. 11, pp. 2099-2109, Nov. 1996.

[17] K. Rambabu and J. Bornemann, "Simplified analysis technique for the initial design of a class of LTCC filters," IEEE Trans. Microw. Theory Tech., vol. 53, no. 5, pp. 1787-1791, May 2005.

[18] J. Uher, J. Bornemann, and U. Rosenberg, Waveguide Components for Antenna Feed Systems-Theory and CAD. Norwood, MA: Artech House, 1993.

[19] A. E. Atia and A. E. Williams, "New type of waveguide bandpass filters for satellite transponders," COMSAT Tech. Rev., vol. 1, no. 1, pp. $21-43,1971$.

[20] M. Mokhtaari, J. Bornemann, and S. Amari, "Advanced filter design using cross-coupled networks with higher-order resonances," in Proc. 35th Eur. Microw. Conf., Paris, France, Oct. 2005, pp. 1423-1426.

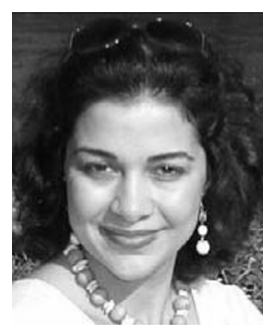

Marjan Mokhtaari received the B.Sc. and M.A.Sc degrees (with honors) from the Sharif University of Technology (SUT), Tehran, Iran, in 1997 and 2000, respectively, and is currently working toward the $\mathrm{Ph} . \mathrm{D}$. degree in advanced microwave filter design at the University of Victoria, Victoria, BC, Canada.

Following a three-year affiliation with the Iran Telecommunication Research Center (ITRC) as a Researcher and RF/Microwave Design Engineer and faculty member, she joined the University of Victoria, in January 2004. Her research interest are passive, active RF, and microwave circuits such as filters, antennas, amplifiers, mixers, numerical, and computational electromagnetics, as well as optimization in microwave theory and inverse scattering.

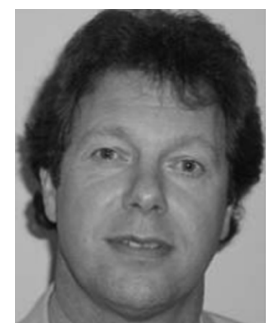

Jens Bornemann (M'87-SM'90-F'02) received the Dipl.-Ing. and the Dr.-Ing. degrees in electrical engineering from the University of Bremen, Bremen, Germany, in 1980 and 1984, respectively.

From 1984 to 1985, he was a Consulting Engineer. In 1985, he joined the University of Bremen, as an Assistant Professor. Since April 1988, he has been with the Department of Electrical and Computer Engineering, University of Victoria, Victoria, BC, Canada, where he became a Professor in 1992. From 1992 to 1995, he was a Fellow with the British Columbia Advanced Systems Institute. In 1996, he was a Visiting Scientist with Spar Aerospace Limited (now MDA Space), Ste-Anne-de-Bellevue, Québec, QC, Canada, and a Visiting Professor with the Microwave Department, University of Ulm, Ulm, Germany. From 1997 to 2002, he was a Co-Director 
with the Center for Advanced Materials and Related Technology (CAMTEC), University of Victoria. In 2003, he was a Visiting Professor with the Laboratory for Electromagnetic Fields and Microwave Electronics, Eidgenössische Technische Hochschule (ETH) Zürich, Zürich, Switzerland. He coauthored Waveguide Components for Antenna Feed Systems. Theory and Design (Artech House, 1993). He has authored/coauthored over 200 technical papers. He is on the Editorial Advisory Boards of the International Journal of Numerical Modelling and the International Journal of Electronics and Communications (AEii). His research activities include RF/wireless/microwave/millimeter-wave components and systems design, and field-theory-based modeling of integrated circuits, feed networks and antennas.

Dr. Bornemann is a Registered Professional Engineer in the Province of British Columbia, Canada. He serves on the Technical Program Committee of the IEEE Microwave Theory and Techniques Society (IEEE MTT-S) International Microwave Symposium (IMS). From 1999 to 2002, he was an associate editor for the IEEE TRANSACTIONS ON MICROWAVE THEORY AND TECHNIQUES in the area of microwave modeling and computer-aided design (CAD).

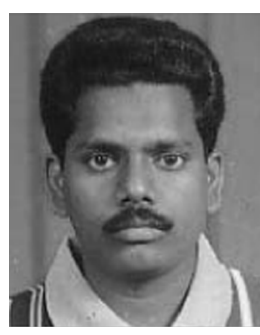

K. Rambabu received the Ph.D. degree from the University of Victoria, Victoria, BC, Canada, in 2004.

He is currently a Research Staff Member with the Institute for Infocomm Research, Singapore. He has authored or coauthored over 40 papers published in refereed journals and conferences. He holds a patent for beam shaping of a cellular base-station antenna. His research interests include design and development of miniaturized passive microwave components and antennas for various applications.

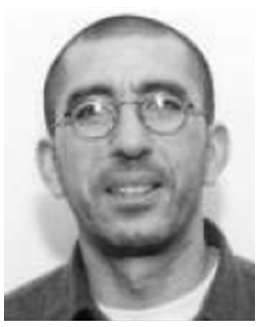

Smain Amari (M'98) received the D.E.S. degree in physics and electronics from Constantine University, Constantine, Algeria, in 1985, and the Masters degree in electrical engineering and $\mathrm{Ph}$.D. degree in physics from Washington University, St. Louis, MO, in 1989 and 1994, respectively.

From 1994 to 2000, he was with the Department of Electrical and Computer Engineering, University of Victoria, Victoria, BC, Canada. From 1997 to 1999, he was a Visiting Scientist with the Swiss Federal Institute of Technology, Zürich, Switzerland, and a Visiting Professor in Summer 2001. Since November 2000, he has been with the Department of Electrical and Computer Engineering, Royal Military College of Canada, Kingston, ON, Canada, where he is currently a Professor. His research interests are numerical analysis, numerical techniques in electromagnetics, applied physics, applied mathematics, wireless and optical communications, computer-aided design (CAD) of microwave components, and application of quantum field theory in quantum many-particle systems. 